\title{
Is human papillomavirus a risk factor for oral squamous cell carcinoma?
}

\author{
Is oral infection with human papillomavirus (HPV) a risk factor for oral \\ squamous cell carcinoma (OSCC)?
}

Miller CS, Johnstone BM. Human papilloma virus as a risk factor for oral squamous cell carcinoma: a meta-analysis, 1982-1997. Oral Surg Oral Med Oral Pathol Oral Radiol Endod 2001; 92:170-179

Data sources Sources used were MEDLINE (January 1980-August 1998) and bibliographies of review articles.

Study selection Studies included were case reports and clinical series that used biochemical, immunological, microscopic or molecular analyses to detect HPV in tissue or cells derived from normal oral mucosa, benign leukoplakia, intraepithelial neoplasia and oral cancer. Data extraction and synthesis The prevalence of HPV determined by various assays was analysed with respect to the clinical and histological nature of the oral lesion. The data were further analysed using a random-effects model. The pooled probability of detecting high-risk versus low-risk HPV genotypes in OSCC was evaluated.

Results The meta-analysis included data from 94 reports (4680 samples). The probability of detecting HPV increased with the increasing dysplastic nature of oral mucosa. Adjustments for assay sensitivity indicated that these estimates may be conservative. The pooled odds ratio from the studies that directly compared prevalence of HPV in oral carcinoma was 5.4, confirming the trend. The pooled probability of detecting high-risk HPV genotypes in OSCC was 2.8-fold greater than that of detecting low-risk HPV (Table 1).

Conclusions These findings provide further evidence that oral infection with HPV, particularly high-risk genotypes, is a significant risk factor for OSCC. Both oral dysplasia and OSCC are more commonly associated with HPV compared with normal oral mucosa.

Table 1. Likelihood of detecting HPV in oral mucosal lesions.

\begin{tabular}{lccr}
\hline Nature of oral epithelium & $\begin{array}{c}\text { Studies/ } \\
\text { datasets } \\
(n / n)^{\mathrm{a}}\end{array}$ & $\begin{array}{c}\text { Likelihood of } \\
\text { detecting } \\
\text { HPV }(\%)\end{array}$ & $\begin{array}{c}95 \% \\
\text { confidence } \\
\text { interval }\end{array}$ \\
\hline Normal oral mucosa & $25 / 27$ & 10 & $6.1-14.6$ \\
Benign leukoplakia & $21 / 22$ & 22.2 & $15.7-29.9$ \\
Intraepithelial neoplasia & $27 / 28$ & 26.2 & $19.6-33.6$ \\
Verrucous carcinoma & $14 / 16$ & 29.5 & $23.0-36.8$ \\
Oral squamous cell carcinoma & $80 / 90$ & 46.5 & $37.6-55.5$ \\
\hline
\end{tabular}

aSome studies used more than one assay to detect human papillomavirus (HPV).

\section{Commentary}

In Western populations, major aetiological factors for the development of intraoral cancer are smoking and alcohol misuse. Several other factors remain speculative and viral infections are included in this group. In the last 20 years, evidence has accumulated regarding the possible association of oncogenic high-risk types of HPV with OSCC. HPV is already implicated in cervical cancer, anorectal cancers and among head and neck cancers for tonsillar carcinoma. ${ }^{1}$ This meta-analysis assessing the risk of HPV status in OSCC and oral premalignant lesions is timely.

Address for correspondence: Craig S Miller, Oral Medicine Section MN 118, Department of Oral Health Practice, University of Kentucky College of Dentistry, 800 Rose Street, Lexington, Kentucky, USA. E-mail: cmiller@pop.uky.edu
The authors carried out a MEDLINE search and hand-searched information using the available review articles. Some grey literature from case reports was included in addition to reported clinical series. The criteria used for tissue sampling and anatomical locations of cancers are clear.

The authors conclude that HPV infection with high-risk genotypes is a significant risk factor for OSCC. In the 94 reports accessed, the incidence of HPV ranged from 0 to $100 \%$. This demonstrates the enormous variation expected in finding HPV in OSCC, largely due to different laboratory techniques employed. In two reports from their own laboratory within a time-period of 1 year the reported detection rate of HPV in OSCC rose from 0\% (when in situ hybridisation was employed) to $67 \%$ (when PCR was utilised). The meta-analysis included assay-sensitivity estimates but these analyses produced results similar to the unadjusted analyses. Further factors to consider in risk assessments are age and population differences. Role of HPV in oral carcinogenesis appears to be minor compared with smoking and alcohol but special population groups may be at risk. For example, $S_{c u l l y}{ }^{2}$ raises the potential for sexual transmission of HPV as an aetiological factor for OSCC in young male adults.

How infection of oral keratinocytes by HPV contributes to malignant transformation has been reviewed. ${ }^{3}$ Oncogenic proteins encoded by E6 and E7 genes from high-risk HPV genotypes (mainly HPV-16 and -18) are able to disrupt cell cycle regulatory proteins such and p53 and pRB by stimulating their degradation. Few studies of HPV E6/E7 gene expression in OSCC ${ }^{4}$ exist, but the theoretical model supports the biological role of HPV. The present article is a valuable contribution, identifying the need for more rigorous research to quantify accurately HPV infection in OSCC. The reviewer is aware of two recent studies, a multicentre study coordinated by the IARC (International Agency for Research on Cancer) and another at John Hopkins University (Baltimore, MD, USA) that may further strengthen our knowledge on the role of HPV in OSCC. Translating these important laboratory findings of HPV infection in neoplasia to therapeutic interventions is already in progress. ${ }^{5}$

\section{Saman Warnakulasuriya}

Department of Oral Medicine, Guy's, King's \& St Thomas' Dental Institute, King's College, London, UK

1. Gillison ML, Koch WM, Capone RB, et al. Evidence for a causal association between human papillomavirus and a subset of head and neck cancers. J Natl Cancer Inst 2000; 92:709-720.

2. Scully $C$. Oral squamous cell carcinoma: from an hypothesis about a virus, to concern about sexual transmission. Oral Oncol 2002; 38:227-234.

3. Sugerman PB, Shillitoe EJ. The high risk human papillomaviruses and oral cancer: evidence for and against a causal relationship. Oral Dis 1997; 3:130-147.

4. Penhallow J, Steingrimsdottir $\mathrm{H}$, Elamin $\mathrm{F}$, et al. p53 alterations and HPV infections are common in oral SCC: p53 gene mutations correlate with the absence of HPV 16-E6 DNA. Int J Oncol 1998; 12:59-68.

5. Yoo SS, Whitmore SE. A human papillomavirus type 16 vaccine. $\mathrm{N}$ Engl J Med 2003; 348:1645-1651.

Evidence-Based Dentistry (2003) 4, 29.

doi:10.1038/sj.ebd.6400171 